\title{
Zero entropy and bounded topology
}

\author{
Gabriel P. Paternain* and Jimmy Petean ${ }^{\dagger}$
}

\begin{abstract}
We study the existence of Riemannian metrics with zero topological entropy on a closed manifold $M$ with infinite fundamental group. We show that such a metric does not exist if there is a finite simply connected $\mathrm{CW}$ complex which maps to $M$ in such a way that the rank of the map induced in the pointed loop space homology grows exponentially. This result allows us to prove in dimensions four and five, that if $M$ admits a metric with zero entropy then its universal covering has the rational homotopy type of a finite elliptic $\mathrm{CW}$ complex. We conjecture that this is the case in every dimension.
\end{abstract}

\section{Introduction}

Let $M^{n}$ be a closed connected smooth manifold. Given a Riemannian metric $g$, let $\phi_{t}$ be the geodesic flow of $g$.

One of the most fundamental dynamical invariants that one can associate to $\phi_{t}$ is the topological entropy, which we denote by $h_{\text {top }}(g)$. It roughly measures the orbit structure complexity of the flow. Positive entropy means in general, that the geodesic flow presents somewhere in the phase space (the unit sphere bundle of the manifold) a complicated dynamical behaviour. There are various equivalent ways of defining entropy, but for the geodesic flow, Mañé's formula [14] provides a clear understanding of this invariant in terms of geodesic arcs. Given points $p$ and $q$ in $M$ and $T>0$, define $n_{T}(p, q)$ to be the number of geodesic arcs joining $p$ and $q$ with length $\leq T$. We have

$$
\mathrm{h}_{\mathrm{top}}(g)=\lim _{T \rightarrow \infty} \frac{1}{T} \log \int_{M \times M} n_{T}(p, q) d p d q .
$$

The main goal of this paper is to address the following natural question: which manifolds admit metrics with zero topological entropy?

A classical result of E. I. Dinaburg [3] asserts that if $M$ admits such a metric, then $\pi_{1}(M)$ must have subexponential growth. It is still unknown if there are finitely presented groups which are of subexponential growth, but not of polynomial growth. If such groups do not exist, then zero entropy implies that $\pi_{1}(M)$ is virtually

\footnotetext{
*G. P. Paternain was partially supported by CIMAT, Guanajuato, México.

$\dagger$ J. Petean is supported by grant 46274-E of CONACYT.
} 
nilpotent, thanks to a celebrated theorem of M. Gromov [8]. For closed geometrizable 3-manifolds, this obstruction on the fundamental group is enough to determine those which admit a metric with zero topological entropy, cf. [1].

In the late 1980's new topological obstructions were found, this time for simply connected manifolds. Y. Yomdin [19] proved a fundamental theorem for general $C^{\infty}$ dynamical systems relating the topological entropy with the volume growth of submanifolds which paved the way to Mañés formula. When combined with the Morse theory of the loop space and a beautiful discovery of Gromov [7] concerning cycles with bounded length in the pointed loop space $\Omega M$, it gave strong restrictions to zero entropy. Namely, if $M$ is simply connected and admits a $C^{\infty}$ metric $g$ with $\mathrm{h}_{\mathrm{top}}(g)=0$, then the sum of the Betti numbers $\sum_{i=1}^{n} \operatorname{dim} H_{i}\left(\Omega M, k_{p}\right)$ grows subexponentially with $n$ for any field of coefficients $k_{p}, p$ prime or zero. When $k_{p}=\mathbb{Q}$, this implies that $M$ is rationally elliptic, i.e. $\pi_{*}(M) \otimes \mathbb{Q}$ is finite dimensional (cf. [6]). We refer to [16] for an account of these developments.

However, these results only hold for simply connected manifolds (or finite $\pi_{1}(M)$ ) because Gromov's theorem does require to control the length of paths running on the 1 -skeleton of a triangulation of $M$. When $M$ is simply connected, one can always collapse the 1-skeleton to a point by a map homotopic to the identity, thus allowing to ignore - at the level of homology - paths running on the 1-skeleton.

What topological restrictions to zero entropy do we have when $\pi_{1}(M)$ is infinite and of subexponential growth? We begun looking at this problem in [18] motivated by the minimal entropy problem for compact complex surfaces. Here we show:

Technical Lemma. Let $M$ be a closed manifold. Let $f: K \rightarrow M$ be a continuous map, where $K$ is a finite simply connected $C W$ complex and let $\Omega(f)$ be the induced map between pointed loop spaces. Let $H_{*}\left(\Omega(f), k_{p}\right)$ be the map induced in homology with some field of coefficients $k_{p}$ and let $R_{i}$ be the rank of this map in dimension $i$. Set

$$
R:=\limsup _{i \rightarrow \infty} \frac{1}{i} \log \left(\sum_{j \leq i} R_{j}\right)
$$

If $R>0$, then given any smooth Riemannian metric $g$ on $M$ we have

$$
\mathrm{h}_{\text {top }}(g)>\frac{\lambda(g)}{2} .
$$

In the inequality, $\lambda(g)$ is the volume entropy of the Riemannian manifold which is defined as the exponential growth rate of the volume of balls in the universal covering of $M$. Recall that Manning's inequality [15] asserts that for any metric $g$, $\mathrm{h}_{\text {top }}(g) \geq \lambda(g)$ and it is well known that $\lambda(g)>0$ if and only if $\pi_{1}(M)$ has exponential growth. We are interested in the inequality as an obstruction to the existence of metrics with vanishing topological entropy, particularly in the case when $\pi_{1}(M)$ has 
subexponential growth. It would be quite interesting to be able to replace $\lambda(g) / 2$ by $\lambda(g)$ in the Technical Lemma.

It seems useful to note the following point: if $\tilde{M}$ is the universal covering of $M$, then the projection induces an isomorphism between the homology of the loop space of $\widetilde{M}$ and the homology of the connected component of the loop space of $M$ given by the contractible loops. Therefore one can consider a CW complex $K$ which maps to $\widetilde{M}$ and then compose with the projection to $M$ to be in the conditions of the Technical Lemma. We will use this remark in all of our examples.

Recall that a connected CW complex $X$ is said to be nilpotent if $\pi_{1}(X)$ is a nilpotent group and operates nilpotently on $\pi_{i}(X)$ for every $i \geq 2$. As an immediate corollary of the lemma we have:

Corollary. Let $M$ be a closed nilpotent manifold. If $M$ admits a smooth metric with zero topological entropy, then $\pi_{*}(\Omega M) \otimes \mathbb{Q}$ is finite dimensional.

Indeed, if $M$ is nilpotent, all the homology groups of $\widetilde{M}$ are finitely generated (cf. [13, Theorem 2.16]) and thus there is a finite simply connected CW complex $K$ and a homotopy equivalence $f: K \rightarrow \widetilde{M}$. The complex $K$ must be rationally elliptic by the Technical Lemma.

We proved the lemma in [18, Theorem C] when $K$ is a smooth compact manifold with boundary which is embedded in $\widetilde{M}$ and for which the corresponding map in the loop space homology is an injection. The disadvantage of this earlier version is that in order to use it we need to have some apriori knowledge of $\widetilde{M}$ so that we can find our embedded $K$, while with the current version $K$ and $f$ will arise by simple topological considerations as in the corollary above.

Nevertheless the old version was good enough to prove results like the following [18, Theorem D]: if $M$ admits a metric with zero entropy and it can be decomposed as $X_{1} \# X_{2}$, where the order of the fundamental group of $X_{1}$ is at least 3, then $X_{2}$ is a homotopy sphere.

We now pose the main topological question that the Technical Lemma suggests:

Question. Let $M$ be a closed manifold whose fundamental group has subexponential growth. If $\operatorname{dim} H_{*}(\widetilde{M}, \mathbb{Q})=\infty$, does there exist a finite 1-connected rationally hyperbolic complex $K$ and a map $f: K \rightarrow M$ for which the rank of $H_{*}(\Omega(f), \mathbb{Q})$ grows exponentially?

Of course one can formulate similar questions for other fields of coefficients, but we believe it should be easier to deal first with the case of characteristic zero, due to the technology at our disposal provided by Rational Homotopy Theory.

If the Question has a positive answer, then the Technical Lemma implies that if $M$ admits a metric with zero entropy then $\widetilde{M}$ has the rational homotopy type of a finite elliptic 1-complex, so we see that zero entropy implies bounded topology in a very strong sense. 
In the present paper we will prove by simple topological arguments that the Question has a positive answer in dimensions 4 and 5. This in turn will give us an essentially complete picture of which 4-manifolds have metrics with zero entropy and will allow us to close some gaps left open in [18].

Let us describe these results in more detail. From now on if in ordinary homology coefficients are not indicated they are meant to be $\mathbb{Z}$. In the next theorem, $\sigma$ and $\chi$ stand for signature and Euler characteristic respectively.

Theorem A. Let $M$ be a closed 4-manifold with infinite fundamental group $\pi$. If $M$ admits a metric with zero topological entropy, then $\sigma(M)=\chi(M)=0, \widetilde{M}$ has the rational homotopy type of a finite simply connected elliptic $C W$ complex and $H_{2}(\tilde{M}) \cong H^{2}(\pi, \mathbb{Z}[\pi])$. Moreover, if we assume further that $\pi$ has polynomial growth then, $M$ is finitely covered by one the following:

(1) $S^{3} \times S^{1}$;

(2) a manifold s-cobordant to $S^{2} \times \mathbb{T}^{2}$;

(3) a nilmanifold.

Using the results on 4-manifolds, we can now complete the classification of compact complex surfaces which admit a metric with zero entropy. We begun this classification in [18], but our results excluded two cases: surfaces of general type and surfaces of class VII with positive second Betti number. It is unknown if there are surfaces of general type homeomorphic to $S^{2} \times S^{2}$ or $\mathbb{C} P^{2} \# \overline{\mathbb{C P}}^{2}$, although it is known that there is no surface of general type diffeomorphic to $S^{2} \times S^{2}$ or $\mathbb{C} P^{2} \# \overline{\mathbb{C P}}^{2}$. We call such a potential example, an exotic surface of general type. In the next theorem we view compact complex surfaces as smooth 4-manifolds and we ignore their complex structures.

Theorem B. Let $S$ be a compact complex surface which is not an exotic surface of general type. Then $S$ admits a metric with zero topological entropy if and only if $S$ is diffeomorphic to one of the following: $\mathbb{C} P^{2}$, a ruled surface of genus 0 or 1 , a complex torus, a hyperelliptic surface, a Hopf surface, a Kodaira surface, or a Kodaira surface modulo a finite group.

Finally in dimension 5 we prove:

Theorem C. Let $M$ be a closed 5-manifold with infinite fundamental group. If $M$ admits a metric with zero entropy, then $\widetilde{M}$ has the rational homotopy type of a finite 1-connected elliptic complex. Moreover, $H_{3}(\tilde{M}) \cong H^{2}(\pi, \mathbb{Z}[\pi])$.

In fact, for most groups $\pi$ with subexponential growth the second end group $H^{2}(\pi, \mathbb{Z}[\pi])$ is either 0 or $\mathbb{Z}$. Our methods also yield information at the torsion level. For example we will show that if $\pi$ has one end and $H^{2}(\pi, \mathbb{Z}[\pi])$ is either 0 or $\mathbb{Z}$, then $H_{2}(\widetilde{M})$ has no finite subgroup as a direct summand. 
In all the known examples of closed manifolds which admit metrics with zero topological entropy, $M$ has a finite covering which is a nilpotent space. The following problem was posed to us by B. Totaro.

Problem. Is it true that if $M$ admits a metric with zero topological entropy, then there is a finite covering of $M$ which is nilpotent?

A positive answer to the Problem implies that the universal covering of $M$ is homotopy equivalent to a finite $\mathrm{CW}$ complex, which by the Technical Lemma must have loop space homology with subexponential growth. Observe that by Theorem A, the Problem has a positive answer in dimension 4 if we assume that $\pi$ has polynomial growth. As Totaro suggested one can now make the following definition of elliptic space to incorporate all these observations and allow infinite fundamental group. A topological space $X$ is elliptic if it is homotopy equivalent to a finite CW complex, it has a finite covering which is a nilpotent space and the loop space homology of the universal covering of $X$ grows polynomially with any field of coefficients. The results in this paper give considerable evidence of the following conjectural fact: If $M$ admits a metric with zero topological entropy it must be elliptic in this broader sense.

Acknowledgements. We thank Burt Totaro for several useful comments on the first draft of the manuscript.

\section{Proof of the Technical Lemma}

Let $(M, g)$ be a Riemannian manifold and let $K$ be a finite simply connected CW complex. Given a continuous map $f: K \rightarrow M$ we let $\Omega(f): \Omega(K) \rightarrow \Omega(M)$ be the obvious map induced between the corresponding pointed loop spaces. The following lemma is essentially due to Gromov [7, 10]:

Lemma 2.1. There exists a constant $C=C(K, f, M, g)$ such that given any homology class $\psi \in H_{i}(\Omega(K))$, the class $\Omega(f)_{*}(\psi)$ can be represented by a cycle of Lipschitz curves in $M$ with length bounded by $C i$.

Proof. Since $K$ is homotopy equivalent to a finite simply connected simplicial complex [11, Theorem 2.C5], we can assume that $K$ is actually a simplicial complex. We can consider $K$ as a subcomplex of a simplex $\Delta^{N}$ and restrict the standard metric on $\Delta^{N}$ to give a metric on $K$. Of course, this metric restricts to the standard Euclidean metric on each simplex of $K$. It is easy to see that one can approximate $f$ by a homotopic map which is Lipschitz with respect to this metric. Therefore we will also assume that the map $f$ is Lipschitz. 
Let $h: L \rightarrow \Omega(K)$ be a map from a finite simplicial complex of dimension $i$ with an $i$-th homology class mapping to $\psi$. The map $h$ corresponds to a map $H: L \times[0,1] \rightarrow K$. Given a positive integer $k$ we will consider the simplicial structure on $[0,1]$ obtained by subdividing the interval into $k$ subintervals of equal length. The simplicial structures on $L$ and $[0,1]$ give a natural cellular decomposition on $L \times[0,1]$.

For this cellular decomposition one can obtain a simplicial approximation similar to the simplicial case as follows:

Let $S$ be any simplicial complex. We call a map $R: L \times[0,1] \rightarrow S$ simplicial if it is a simplicial map when restricted to each $L \times\{j / k\}$ and for any $x \in L, R(x, j / k)$ and $R(x,(j+1) / k)$ belong to a simplex in $S$ and the restriction of $R$ to the vertical segment $\{x\} \times[j / k,(j+1) / k]$ is linear.

Given a continuous map $r: L \times[0,1] \rightarrow S$, we say that $R$ is a simplicial approximation of $r$ if it is a simplicial map such that $R(q) \in \operatorname{Carrier}(r(q))$ (the smallest simplex containing $r(q))$ for any $q \in L \times[0,1]$. It is easy to see as in the simplicial case that if $R$ is a simplicial approximation of $r$, then $R$ and $r$ are homotopic.

Recall now that the open star of a vertex in a simplicial complex is the union of the interior of all the simplices containing the vertex, and for a vertex $(v, j / k) \in L \times[0,1]$ define its open star as $\operatorname{Star}(v, j / k)=\operatorname{Star}(v) \times((j-1) / k,(j+1) / k)$. It is clear that after enough subdivisions of the simplicial structure on $L$ and taking $k$ big enough, we can assume that the diameter of the open star of any vertex is as small as we want. Therefore we can assume that for any vertex $(v, j / k) \in L \times[0,1]$ there exists a vertex $w \in S$ such that the open star of $(v, j / k)$ is contained in $r^{-1}(\operatorname{Star}(w))$. We define $R(v, j / k)=w$. As in the simplicial case, we can extend $R$ to each $L \times\{j / k\}$ as a simplicial approximation of the restriction of $r$ to $L \times\{j / k\}$.

Given $x \in L$, let $\sigma=\operatorname{Carrier}(x)$ and let $v_{1}, \ldots, v_{l}$ be the vertices of $\sigma$. Let $w_{i}=$ $R\left(v_{i}, j / k\right)$ and $w_{i+l}=R\left(v_{i},(j+1) / k\right)$. Note that Interior $(\sigma) \times(j / k,(j+1) / k)$ is contained in the open stars of each $\left(v_{i}, m / k\right)(m=j$ or $j+1)$. By construction we have that $r(\operatorname{Interior}(\sigma) \times(j / k,(j+1) / k))$ is contained in $\operatorname{Star}\left(w_{i}\right)$ for each $i=1, \ldots, 2 l$. The intersections of these sets is nonempty and this implies that those vertices form a simplex in $S$. We have done this to show that there exists a simplex of $S$ which contains both $R(x, j / k)$ and $R(x,(j+1) / k)$ and so we can extend $R$ as a simplicial map on $L \times[0,1]$.

Let us finally check that $R$ is actually a simplicial approximation of $r$. We have to show that for any $(x, t) \in L \times[0,1], R(x, t) \in \operatorname{Carrier}(r(x, t))$. We already know this if $t$ is a vertex of [0,1]. So we can assume that $t \in(j / k,(j+1) / k)$ for some $j$. As in the previous paragraph, let $v_{1}, \ldots, v_{l}$ be the vertices of Carrier $(x)$ and let $w_{1}, \ldots, w_{2 l}$ be the corresponding vertices in $S$. We have that $(x, t) \in \operatorname{Star}\left(v_{i}, j / k\right) \cap$ $\operatorname{Star}\left(v_{i},(j+1) / k\right)$ for each $i=1, \ldots, l$. Then $r(x, t)$ lies in $r\left(\operatorname{Star}\left(v_{i}, j / k\right) \cap\right.$ $\left.\operatorname{Star}\left(v_{i},(j+1) / k\right)\right) \subset \operatorname{Star}\left(w_{i}\right) \cap \operatorname{Star}\left(w_{i+l}\right)$. This implies that $w_{i}, w_{i+l}$ are elements of Carrier $(r(x, t))$. Since $R(x, t)$ is a linear combination of $w_{1}, \ldots, w_{2 l}$ and all these 
vertices belong to the simplex $\operatorname{Carrier}(r(x, t))$, we get that $R(x, t) \in \operatorname{Carrier}(r(x, t))$ and therefore $R$ is a simplicial approximation of $r$.

Hence we can take a simplicial approximation $R$ of the map $H: L \times[0,1] \rightarrow K$. It is clear from the discussion above that we may assume that $R$ fixes the end points and thus it can be viewed also as a map $r: L \rightarrow \Omega(K)$. Let us consider the space $\Omega(K)_{p l, k} \subset \Omega(K)$ given by those paths which are linear on each segment of the form $[j / k,(j+1) / k]$. Note that by construction there exists a $k$ such that the image of $r$ is contained in $\Omega(K)_{p l, k}$. Each element in $\Omega(K)_{p l, k}$ determines a point in $K^{k-1}$ and in this way we identify $\Omega(K)_{p l, k}$ with a subset of $K^{k-1}$ (recall that the initial and final points are fixed). The simplicial structure on $K$ induces a cellular decomposition in $K^{k-1}$ and $\Omega(K)_{p l, k}$ is a subcomplex: it is the union of all the cells $\sigma_{1} \times \cdots \times \sigma_{k-1}$ such that $\sigma_{i}$ and $\sigma_{i+1}$ are contained in a simplex of $K$ and the same for the initial point and $\sigma_{1}$ and the end point and $\sigma_{k-1}$.

After another homotopy we can assume that the image of $r$ is contained in the $i$-th skeleton of $\Omega(K)_{p l, k}$ with respect to the cell decomposition described above.

Since $K$ is simply connected there exists a Lipschitz map $\alpha: K \rightarrow K$ homotopic to the identity, which maps the whole 1-skeleton of $K$ to a point.

Now let $c$ be a path in the $i$-th skeleton of $\Omega(K)_{p l, k}$. This means that $c$ belongs to a cell of the form $\sigma_{1} \times \cdots \times \sigma_{k-1}$ with $\Sigma_{j} \operatorname{dim}\left(\sigma_{j}\right) \leq i$. Thus, the path $c$ is formed by segments joining a pair of vertices of the triangulation and at most $2 i$ segments in which one of the points is not a vertex. After composing with the map $\alpha$ the former are sent to a point while the latter are sent to paths of length bounded by a constant which depends on $\alpha$ but not on $i$. Hence there exists a constant $C^{\prime}$ such that the image of the $i$-th skeleton of $\Omega(K)_{p l, k}$ is sent by composition with $\alpha$ to a set of paths with length bounded by $C^{\prime} i$. In this way we see that we can represent $\psi$ by a cycle formed with paths with length bounded by $C^{\prime} i$. Composing with $f$ we see that we can represent $\Omega(f)_{*}(\psi)$ by a cycle formed with paths of length bounded by $C i$, where $C$ is a constant depending only on $\alpha$ and the Lipschitz constant of $f$.

We will use the lemma in the form of the following corollary.

Corollary 2.2. Let $(N, g)$ be a connected complete Riemannian manifold. Let $K$ be a finite simply connected $C W$ complex and $f: K \rightarrow N$ be a continuous map. Denote by $H_{i}\left(\Omega(f), k_{p}\right)$ the induced map between the $i$-th homology groups of the corresponding pointed loop spaces (for some field of coefficients $k_{p}$ ) and let $R_{i}$ be the rank of this map. Then, there exists a positive constant $C$ depending only on $K, f, g$ such that for any $x \in K, T \geq C i$ and any $y \in B(f(x), T / 2)$ we have that $b_{i}\left(\Omega^{T}(N, f(x), y), k_{p}\right) \geq R_{i}$.

Proof. We know from the lemma that for any $i$-th homology class $\psi$ in $\Omega(K, x, x)$, $\Omega(f)_{*}(\psi)$ can be represented by a cycle in $\Omega^{C^{\prime} i}(N, f(x), f(x))$. Consider now a minimizing geodesic between $f(x)$ and $y$. Following the paths in the cycle by this 
geodesic we obtain a cycle in $\Omega^{C^{\prime} i+d(f(x), y)}(N, f(x), y)$. If $C=2 C^{\prime}, T \geq C i$ and $y \in B(f(x), T / 2)$ we get that our new cycle is in $\Omega^{T}(N, f(x), y)$ and the corollary follows.

We are now ready to prove the Technical Lemma in the introduction.

Technical Lemma. Let $M$ be a closed manifold. Let $f: K \rightarrow M$ be a continuous map, where $K$ is a finite simply connected $C W$ complex and let $\Omega(f)$ be the induced map between pointed loop spaces. Let $H_{*}\left(\Omega(f), k_{p}\right)$ be the map induced in homology with some field of coefficients $k_{p}$ and let $R_{i}$ be the rank of this map in dimension $i$. Set

$$
R:=\limsup _{i \rightarrow \infty} \frac{1}{i} \log \left(\sum_{j \leq i} R_{j}\right) .
$$

If $R>0$, then given any smooth Riemannian metric $g$ on $M$ we have

$$
\mathrm{h}_{\mathrm{top}}(g)>\frac{\lambda(g)}{2} \text {. }
$$

Proof. By the lifting property of covering spaces we can assume that we have a map $f: K \rightarrow \widetilde{M}$. Let us recall that for any $x \in M$ (cf. [16]),

$$
\mathrm{h}_{\text {top }}(g) \geq \limsup _{T \rightarrow \infty} \frac{1}{T} \log \int_{M} n_{T}(x, y) d y .
$$

Let $p: \tilde{M} \rightarrow M$ be the covering projection. It is easy to check that given any $x \in \widetilde{M}$ we have

$$
\int_{M} n_{T}(p(x), y) d y=\int_{\widetilde{M}} n_{T}(x, y) d y=\int_{B(x, T)} n_{T}(x, y) d y .
$$

Thus for any $x \in \tilde{M}$ we have

$$
\mathrm{h}_{\text {top }}(g) \geq \limsup _{T \rightarrow \infty} \frac{1}{T} \log \int_{B(x, T)} n_{T}(x, y) d y .
$$

Now assume that $x=f(z)$ for some $z \in K$. Morse theory tells us that if $y$ and $x$ are not conjugate, then

$$
n_{T}(x, y) \geq \sum_{j \geq 0} b_{j}\left(\Omega^{T}(\tilde{M}, x, y), k_{p}\right) .
$$

But now, using the previous corollary we get that if $y \in B(x, C i / 2)$,

$$
\sum_{j \geq 0} b_{j}\left(\Omega^{C i}(\tilde{M}, x, y), k_{p}\right) \geq \sum_{j \leq i} R_{j},
$$


where $C$ is the constant appearing in the corollary. Integrating the previous inequalities with respect to $y \in B(x, C i / 2)$ yields:

$$
\int_{B(x, C i / 2)} n_{C i}(x, y) d y \geq\left(\sum_{j \leq i} R_{j}\right) \operatorname{Vol}(B(x, C i / 2)) .
$$

Therefore we obtain

$$
\begin{aligned}
\mathrm{h}_{\mathrm{top}}(g) & \geq \limsup _{i \rightarrow \infty} \frac{1}{C i} \log \int_{B(x, C i)} n_{C i}(x, y) d y \\
& \geq \limsup _{i \rightarrow \infty} \frac{1}{C i} \log \left(\sum_{j \leq i} R_{j}\right) \operatorname{Vol}(B(x, C i / 2)) .
\end{aligned}
$$

And thus, if $R$ is the exponential growth rate of $\sum_{j \leq i} R_{j}$,

$$
\mathrm{h}_{\text {top }}(g) \geq \frac{R}{C}+\frac{\lambda(g)}{2} \text {. }
$$

\section{Topological preliminaries}

In the following sections we will try to apply the Technical Lemma to find obstructions to zero entropy. This is of course a purely topological problem and in this section we will summarize some general techniques and concepts we will use.

3.1. Domination. Recall that a topological space $Z$ is dominated by a topological space $Y$ if there exist continuous maps $r: Y \rightarrow Z$ and $\iota: Z \rightarrow Y$ such that $r \iota$ is homotopic to the identity of $Z$. In several occasions we will make use of the following lemma, sometimes without explicit mention to it.

Lemma 3.1. Let $X$ and $Y$ be simply connected spaces which are rational homotopy equivalent. Suppose $Z$ is dominated by $Y$. Then there is a map $g: Z \rightarrow X$ such that

$$
H_{*}(\Omega(g), \mathbb{Q}): H_{*}(\Omega(Z), \mathbb{Q}) \rightarrow H_{*}(\Omega(X), \mathbb{Q})
$$

is an injection.

Proof. Let $f: Y \rightarrow X$ be a rational homotopy equivalence. Since $Y$ dominates $Z$, there is a map $\iota: Z \rightarrow Y$ such that $H_{*}(\Omega(\iota), \mathbb{Q}): H_{*}(\Omega(Z), \mathbb{Q}) \rightarrow H_{*}(\Omega(Y), \mathbb{Q})$ is an injection. By the Whitehead-Serre theorem (see Theorem 8.6 in [6]), $H_{*}(\Omega(f), \mathbb{Q}): H_{*}(\Omega(Y), \mathbb{Q}) \rightarrow H_{*}(\Omega(X), \mathbb{Q})$ is an isomorphism and therefore $g:=f \circ \iota$ has the desired property. 
3.2. Moore spaces. Given $G$ an abelian group, let $M(G, n), n>1$, be the Moore space (uniquely determined up to homotopy type) whose $n$-th homology group is $G$ and whose $i$-th homology group is zero for $i \neq n$. For example, $M(\mathbb{Z}, n)=S^{n}$ and $M\left(\mathbb{Z}_{m}, n\right)=e^{n+1} \cup_{f} S^{n}$, where $f: S^{n} \rightarrow S^{n}$ is a map of degree $m$. Note that $M(G, n)$ has the rational homotopy type of a wedge of spheres.

We will use the following properties of Moore spaces (cf. [2, Proposition 1.7]):

(1) $M(A \oplus B, n)=M(A, n) \vee M(B, n)$;

(2) a morphism $f: A \rightarrow B$ induces a continuous map $M(f): M(A, n) \rightarrow M(B, n)$, so that $M(f g)=M(f) M(g)$ and $M(f)_{*}=f$.

In particular if $f: A \rightarrow B$ is an injection and there exists $g: B \rightarrow A$ such that $g f=1_{A}$ then $M(B, n)$ dominates $M(A, n)$. If $G$ is a finitely generated abelian group, then $M(G, n)$ is given by the wedge sum of copies of $S^{n}$ and copies of $M\left(\mathbb{Z}_{m}, n\right)$ and $M(G, n)$ dominates any of these Moore subspaces.

3.3. Homology decompositions. Every simply connected $C W$ complex $Y$ has a homology decomposition (cf. Theorem 4H.3 in [11] or [2, Theorem 2.2]). This means that there exists a homotopy equivalence $f: X \rightarrow Y$ such that $X$ can be constructed by the following iterated procedure.

Let $G_{n}:=H_{n}(Y)$. There is an increasing sequence of complexes $X_{1} \subset X_{2} \subset \cdots$ with $H_{i}\left(X_{n}\right)=G_{i}$ for $i \leq n$ and $H_{i}\left(X_{n}\right)=0$ for $i>n$ where:

(1) $X_{1}$ is a point and $X_{2}$ is the Moore space $M\left(G_{2}, 2\right)$;

(2) $X_{n+1}$ is the mapping cone of a cellular map $h_{n}: M\left(G_{n+1}, n\right) \rightarrow X_{n}$ such that the induced map $\left(h_{n}\right)_{*}: H_{n}\left(M\left(G_{n+1}, n\right)\right) \rightarrow H_{n}\left(X_{n}\right)$ is trivial;

(3) $X=\bigcup_{n} X_{n}$.

If $Y$ is a simply connected $\mathrm{CW}$ complex whose only non-zero homology groups are $H_{n}(Y)$ and $H_{n+1}(Y)$, and $H_{n+1}(Y)$ is a free abelian group, the homology decomposition says in this case that $Y$ has the homotopy type of $M\left(G_{n}, n\right) \vee M\left(G_{n+1}, n+1\right)$ (cf. for example [2, Lemma 2.6.5]). From this we deduce the next result.

Proposition 3.2. Suppose $Y$ is a simply connected $C W$ complex whose only non-zero homology groups are $H_{n}(Y)$ and $H_{n+1}(Y)$, where $H_{n+1}(Y)$ is a free abelian group. Then $Y$ dominates $M\left(H_{n}(Y), n\right)$ and $M\left(H_{n+1}(Y), n+1\right)$.

Remark 3.3. An important point in the proofs of Theorems $\mathrm{A}$ and $\mathrm{C}$ will be the fact that the rational Hurewicz map $\pi_{*}(\widetilde{M}) \otimes \mathbb{Q} \rightarrow H_{+}(\widetilde{M}, \mathbb{Q})$ is surjective.

Let $X$ be an arbitrary simply connected CW complex. By Theorem 4.5 in [6] the property of $\pi_{*}(X) \otimes \mathbb{Q} \rightarrow H_{+}(X, \mathbb{Q})$ being surjective is equivalent to saying that $X$ has the rational homotopy type of a wedge of spheres or that $X$ has the rational homotopy type of a suspension. 
Proposition 3.4. Suppose $X$ is a simply connected $C W$ complex whose only non-zero homology groups are $H_{n}(X)$ and $H_{n+1}(X)$.

(1) If $b_{n}:=\operatorname{dim} H_{n}(X, \mathbb{Q}) \geq 2$ there exists a map $g: S^{n} \vee S^{n} \rightarrow X$ such that $H_{*}(\Omega(g), \mathbb{Q})$ is an injection.

(2) If $b_{n} \geq 1$ and $H_{n+1}(X)=\mathbb{Z}$, then there exists a map $g: S^{n} \vee S^{n+1} \rightarrow X$ such that $H_{*}(\Omega(g), \mathbb{Q})$ is an injection.

Proof. Note that the Hurewicz map $\pi_{*}(X) \rightarrow H_{+}(X)$ is surjective. Hence $X$ has the rational homotopy type of a wedge of spheres and by Lemma 3.1, if $b_{n} \geq 2$ there exists a map $g: S^{n} \vee S^{n} \rightarrow X$ such that $H_{*}(\Omega(g), \mathbb{Q})$ is an injection which proves the first item. Similarly, if $b_{n} \geq 1$ and $H_{n+1}(X)=\mathbb{Z}$, then Lemma 3.1 gives a map $g: S^{n} \vee S^{n+1} \rightarrow X$ such that $H_{*}(\Omega(g), \mathbb{Q})$ is an injection, which proves the second item.

3.4. Ends of groups, $\ell_{2}$-Betti numbers and amenability. The space of ends $E(X)$ of a locally compact separable metric space $X$ is given by the inverse limit

$$
\lim _{K \subset X} \pi_{0}(X-K),
$$

where the sets $K$ are compact. The space $E(X)$ is a totally disconnected topological space and when $X$ is connected and locally connected, $E(X)$ is compact. Given a group $\pi$ acting freely on a connected simplicial complex $X$ with finite quotient, the homeomorphism type of $E(X)$ only depends on $\pi$. The cardinality of $E(X)$ is usually denoted by $e(\pi)$ and is called number of ends of $\pi$. A finitely generated group $\pi$ has $0,1,2$ or infinitely many ends. It has 0 ends if and only if it is finite.

The higher order end groups of a group $\pi$ are defined as the cohomology groups of a $K(\pi, 1)$ space with coefficients in the group ring $\mathbb{Z}[\pi]$. We denote them by $H^{k}(\pi, \mathbb{Z}[\pi]), k \geq 0$. If the group is infinite, then $H^{1}(\pi, \mathbb{Z}[\pi])$ is a free abelian group of rank $e(\pi)-1$.

The group $\pi$ has two ends if and only if it is virtually $\mathbb{Z}$. If $\pi$ has infinitely many ends, then it must contain a non-cyclic free subgroup by virtue of Stallings' structure theorem.

Finally, we note that if $\tilde{M}$ is the universal covering of a closed $n$-dimensional manifold $M$ with infinite fundamental group $\pi$, then $H^{1}(\pi, \mathbb{Z}[\pi]) \cong H_{n-1}(\tilde{M})$. Of course, one also has $H_{n}(\tilde{M})=0$.

Let $M$ be a closed manifold with an infinite amenable fundamental group and let $\beta_{k}^{(2)}$ be the $k$-th $\ell_{2}$-Betti number of the universal covering of $M$. It is interesting to note the following fact:

- If $\operatorname{dim} H_{k}(\tilde{M}, \mathbb{Q})$ is finite, then $\beta_{k}^{(2)}=0$ ([4]). 
This prompts the following question which is closely related to the Question in the introduction: If for some $k, \beta_{k}^{(2)} \neq 0$, is it true that there exists a finite 1-connected rationally hyperbolic complex $K$ and a map $f: K \rightarrow \widetilde{M}$ for which the rank of $H_{*}(\Omega(f), \mathbb{Q})$ grows exponentially?

We will make use of the following theorem.

Theorem 3.5. Let $M$ be a closed $n$-manifold with an infinite amenable fundamental group $\pi$ and let $\widetilde{M}$ be the universal covering of $M$. Suppose $\tilde{M}$ is $(k-1)$-connected, $k \geq 2$, and $\operatorname{dim} H_{k}(\widetilde{M}, \mathbb{Q})$ is finite. Then $H_{n-k}(\widetilde{M}) \cong H^{k}(\pi, \mathbb{Z}[\pi])$.

Proof. The proof is exactly the same as the proof of Theorem 3.1 in [5]. The hypothesis $\operatorname{dim} H_{k}(\widetilde{M}, \mathbb{Q})<\infty$ and the amenability of $\pi$ ensures that the $k$-th $\ell_{2}$-Betti number vanishes and one argues with the commutative diagram on page 507 to conclude that $H_{\text {comp }}^{k}(\widetilde{M}, \mathbb{Z}) \cong H^{k}(\pi, \mathbb{Z}[\pi])$. By Poincaré duality $H_{n-k}(\widetilde{M}) \cong H_{\text {comp }}^{k}(\widetilde{M}, \mathbb{Z})$.

\section{Proof of Theorems A and B}

We first show:

Theorem 4.1. Let $M$ be a closed 4-manifold with infinite fundamental group $\pi$. If $M$ admits a Riemannian metric with zero topological entropy, then $\chi(M)=\sigma(M)=0$ and $H_{2}(\widetilde{M}) \cong H^{2}(\pi, \mathbb{Z}[\pi])$. Moreover, $\widetilde{M}$ has the rational homotopy type of a point, $S^{2}$ or $S^{3}$.

Proof. If $M$ admits a metric with zero entropy, $\pi$ has subexponential growth and hence it is amenable and can only have 1 or 2 ends. Following B. Eckmann in [4], we note that if $\pi$ is amenable we can construct a Følner sequence, that is, an increasing sequence $Y_{j}, j=1,2,3, \ldots$, of finite subcomplexes of $\widetilde{M}$ with the following properties:

(1) $Y_{j}$ consists of $N_{j}$ translates of a closed cellular fundamental domain $D$ for the action of $\pi$

(2) $\bigcup_{j} Y_{j}=\tilde{M}$;

(3) let $\dot{N}_{j}$ be the number of translates of $D$ which meet the topological boundary of $Y_{j}$; then

$$
\lim _{j \rightarrow \infty} \frac{\dot{N}_{j}}{N_{j}}=0 .
$$

Eckmann shows in [4, p. 389] that

$$
\chi(M)=\lim _{j \rightarrow \infty} \frac{b_{2}\left(Y_{j}\right)}{N_{j}}
$$


where $b_{2}\left(Y_{j}\right)=\operatorname{dim} H_{2}\left(Y_{j}, \mathbb{Q}\right)$. Moreover, by Proposition 2.1 in [4] we know that if $b_{2}(\tilde{M})$ is finite, we must have $\lim _{j \rightarrow \infty} \frac{b_{2}\left(Y_{j}\right)}{N_{j}}=0$.

Note that $H_{1}(\tilde{M})=H_{4}(\tilde{M})=0$ and hence the Hurewicz map $\pi_{*}(\tilde{M}) \rightarrow H_{+}(\tilde{M})$ is onto and $\widetilde{M}$ has the rational homotopy type of a wedge of spheres

$$
X:=\left(\vee_{\alpha} S_{\alpha}^{2}\right) \vee\left(\vee_{\beta} S_{\beta}^{3}\right)
$$

Since $X$ dominates any finite subcollection of them and the rational loop space homology of the wedge of at least two spheres grows exponentially, the Technical Lemma implies that $\widetilde{M}$ must have the rational homotopy type of either a point, $S^{2}$ or $S^{3}$. Thus $b_{2}(\tilde{M})$ is finite and $\chi(M)=0$ as desired.

To prove that $\sigma(M)=0$, we use the following observation of Gromov in [9, p. 85]: if $\sigma(M) \neq 0$, then $b_{2}(\tilde{M})$ must be infinite (this is a consequence of the amenability of $\pi$ and the index theorem for infinite coverings).

Once we know $\chi(M)=0$, the isomorphism between $H_{2}(\tilde{M})$ and $H^{2}(\pi, \mathbb{Z}[\pi])$ is precisely Theorem 3.1 in [5] or Theorem 3.5.

Remark 4.2. Let $M$ be a closed manifold of dimension $2 k$ and suppose that $\pi_{i}(M)=$ 0 for $1<i \leq k-1$ (the condition is vacuous for $k=2$ ). Suppose further that $\pi:=$ $\pi_{1}(M)$ satisfies the following property: it is infinite and the end groups $H^{i}(\pi, \mathbb{Z}[\pi])$ are zero for $0<i<k$. It is quite easy to check (see Proposition 2.1 in [5]) that $H_{i}(\widetilde{M}) \cong H^{2 k-i}(\pi, \mathbb{Z}[\pi])$ for $k<i \leq 2 k$. Thus $H_{k}(\widetilde{M})$ is the only non-zero homology group. If $M$ admits a metric with zero entropy, then $\chi(M)=0$. The proof is the same as in the 4-dimensional case.

Corollary 4.3. Let $M$ be a closed 4-manifold whose fundamental group has two ends. If $M$ admits a metric with zero topological entropy, it is finitely covered by $S^{3} \times S^{1}$.

Proof. Theorem 11.1 in [12] says that a closed 4-manifold whose fundamental group has two ends and $\chi(M)=0$ is finitely covered by $S^{3} \times S^{1}$.

Finding the homeomorphism types of such manifolds is a fairly complicated problem, we refer the interested reader to Chapter 11 in [12].

The last corollary and Theorem 4.1 tell us that if we wish to move further into the classification of closed 4-manifolds which admit a metric of zero entropy we need to know more about $H^{2}(\pi, \mathbb{Z}[\pi])$ for $\pi$ with subexponential growth and one end. As far as we know, there is no general result in this direction. However, note that if $\pi$ is the fundamental group of a closed manifold whose universal covering is $\mathbb{R}^{n}$, then $H^{2}(\pi, \mathbb{Z}[\pi])$ is zero if $n \neq 2$ and $\mathbb{Z}$ if $n=2$.

We can state: 
Corollary 4.4. Let $M$ be a closed 4-manifold with $H^{2}(\pi, \mathbb{Z}[\pi])=\mathbb{Z}$. If $M$ admits a metric with zero topological entropy, $M$ has a covering space of degree dividing 4 which is s-cobordant to $S^{2} \times \mathbb{T}^{2}$.

Proof. By Theorem $4.1, \pi_{2}(M) \cong H_{2}(\tilde{M}) \cong H^{2}(\pi, \mathbb{Z}[\pi])=\mathbb{Z}$. The corollary now follows from Theorem 10.1 in [12].

There is no example known of a finitely presented group which is of subexponential growth, but not of polynomial growth. Recall that the existence of a metric with zero entropy implies subexponential growth of $\pi$.

Theorem 4.5. Let $M$ be a closed 4-manifold whose fundamental group $\pi$ is infinite and has polynomial growth. If $M$ admits a metric with zero topological entropy, then $M$ is finitely covered by one of the following:

(1) $S^{3} \times S^{1}$;

(2) a manifold s-cobordant to $S^{2} \times \mathbb{T}^{2}$;

(3) a nilmanifold.

Proof. By a celebrated theorem of Gromov, $\pi$ is virtually nilpotent. Thus by passing to a finite covering we can assume that $\pi$ is nilpotent. It follows that $\pi$ coincides with its Hirsch-Plotkin radical $\sqrt{\pi}$, which is the maximal nilpotent normal subgroup.

Let $h(\pi)$ denote the Hirsch length of $\pi$, i.e., the number of cyclic factors of a composition series. If $h(\pi) \leq 2$, then up to finite index, $\pi$ must be $\mathbb{Z}$ or $\mathbb{Z}^{2}$. If $\pi$ is $\mathbb{Z}$, then by Corollary 4.3, $M$ falls under item 1 . Similarly, if $\pi$ is $\mathbb{Z}^{2}$, by Corollary 4.4, $M$ falls under item 2.

Finally if $h(\pi)=h(\sqrt{\pi}) \geq 3$, Corollary 8.1.1 in [12] implies that $M$ is finitely covered by a nilmanifold, since by Theorem $4.1, \chi(M)=0$.

Theorems 4.1 and 4.5 give Theorem A.

4.1. Compact complex surfaces. Using the results on 4-manifolds from the previous subsection, we can now complete the classification of compact complex surfaces which admit a metric with zero entropy. We begun this classification in [18], but our results excluded two cases:

(1) surfaces of general type;

(2) surfaces of class VII with positive second Betti number.

Recall that a compact complex surface is of general type if it has Kodaira dimension 2. The surface is said to be of class VII if it has Kodaira dimension $-\infty$ and first Betti number equal to 1 . More precisely, in [18, Theorems E and F] we proved: 
Theorem 4.6. Let $S$ be a compact complex surface which is not of general type. Moreover, suppose that $S$ is not a surface of class VII with positive second Betti number. Then $S$ admits a metric with zero topological entropy if and only if $S$ is diffeomorphic to one of the following: $\mathbb{C} P^{2}$, a ruled surface of genus 0 or 1 , a complex torus, a hyperelliptic surface, a Hopf surface, a Kodaira surface, or a Kodaira surface modulo a finite group.

It is well known that surfaces of general type have $\chi>0$. A surface of class VII has first Betti number equal to one, thus a surface of class VII with positive second Betti number must also have $\chi>0$. Hence in both cases, Theorem 4.1 implies that neither of these classes admits a metric of zero entropy unless the fundamental group is finite. Obviously, surfaces of class VII have an infinite fundamental group. On the other hand, we know that a closed simply connected 4-manifold that admits a metric with zero entropy must be homeomorphic to $S^{4}, \mathbb{C} P^{2}, S^{2} \times S^{2}, \mathbb{C} P^{2} \# \overline{\mathbb{C P}}^{2}$ or $\mathbb{C} P^{2} \# \mathbb{C} P^{2}$ (cf. [17]). Thus if there exists a surface of general type with a metric of zero entropy and finite fundamental group, its universal covering (which is also a surface of general type) would have to be homeomorphic to $S^{2} \times S^{2}$ or $\mathbb{C} P^{2} \# \overline{\mathbb{C P}}^{2}$. It is unknown whether there are such exotic examples, although it is known that there is no surface of general type diffeomorphic to $S^{2} \times S^{2}$ or $\mathbb{C} P^{2} \# \overline{\mathbb{C P}}^{2}$. Below we call such a potential example, an exotic surface of general type.

We now combine this discussion with Theorem 4.6 to obtain:

Theorem B. Let $S$ be a compact complex surface which is not an exotic surface of general type. Then $S$ admits a metric with zero topological entropy if and only if $S$ is diffeomorphic to one of the following: $\mathbb{C} P^{2}$, a ruled surface of genus 0 or 1 , a complex torus, a hyperelliptic surface, a Hopf surface, a Kodaira surface, or a Kodaira surface modulo a finite group.

\section{Proof of Theorem $C$}

Theorem C. Let $M$ be a closed 5-manifold with infinite fundamental group. If $M$ admits a metric with zero entropy, then $\widetilde{M}$ has the rational homotopy type of a finite 1-connected elliptic complex. Moreover, $H_{3}(\tilde{M}) \cong H^{2}(\pi, \mathbb{Z}[\pi])$.

Proof. We know that since $\pi$ is infinite amenable it can only have one or two ends. Moreover, we know that $H_{5}(\tilde{M})=0$ and $H_{4}(\tilde{M}) \cong H^{1}(\pi, \mathbb{Z}[\pi])$.

Suppose first that $\pi$ has one end. Since $H_{4}(\tilde{M})=0$, the Hurewicz map $\pi_{*}(\tilde{M}) \rightarrow$ $H_{+}(\widetilde{M})$ is surjective and $\widetilde{M}$ has the rational homotopy type of a wedge of spheres

$$
X:=\left(\vee_{\alpha} S_{\alpha}^{2}\right) \vee\left(\vee_{\beta} S_{\beta}^{3}\right)
$$


Since $X$ dominates any finite wedge $K$ of spheres from the collection of $S_{\alpha}^{2}$ and $S_{\beta}^{3}$, we conclude that if $\operatorname{dim} H_{+}(\tilde{M}, \mathbb{Q}) \geq 2$, there exist a finite simply connected rationally hyperbolic complex $K$ and a map $f: K \rightarrow \widetilde{M}$ such that $H_{*}(\Omega(f), \mathbb{Q})$ is injective. Hence the Technical Lemma implies that $\operatorname{dim} H_{+}(\widetilde{M}, \mathbb{Q}) \leq 1$ and $\widetilde{M}$ has the rational homotopy type of a point, $S^{2}$ or $S^{3}$.

Suppose now that $\pi$ has two ends and so $H_{4}(\tilde{M})=\mathbb{Z}$. Let us consider the homology decomposition of $\widetilde{M}$ and note that $\widetilde{M}$ has the homotopy type of the mapping cone of a cellular map $h_{3}: M(\mathbb{Z}, 3) \rightarrow X_{3}$ such that $\left(h_{3}\right)_{*}: H_{3}(M(\mathbb{Z}, 3)) \rightarrow H_{3}\left(X_{3}\right)$ is trivial. Since the Hurewicz map $\pi_{*}\left(X_{3}\right) \rightarrow H_{+}\left(X_{3}\right)$ is onto, $X_{3}$ has the rational homotopy type of a wedge of spheres

$$
Y:=\left(\vee_{\alpha} S_{\alpha}^{2}\right) \vee\left(\vee_{\beta} S_{\beta}^{3}\right)
$$

and let $f: X_{3} \rightarrow Y$ be a rational homotopy equivalence. The image of the map $f \circ h_{3}$ can only intersect a finite number of spheres from the collection. Therefore if the collection is infinite it would follow that $\widetilde{M}$ has the rational homotopy type of a space that dominates the wedge product of two spheres. Since the rational loop space homology of the wedge of two spheres grows exponentially the Technical Lemma would imply positive entropy. Therefore there are only a finite number of 2 -spheres and 3-spheres in the collection and $\widetilde{M}$ has the rational homotopy type of a finite $\mathrm{CW}$ complex (which of course must be elliptic).

The conclusion $H_{3}(\widetilde{M}) \cong H^{2}(\pi, \mathbb{Z}[\pi])$ follows now directly from Theorem 3.5.

\subsection{Torsion of 5-manifolds}

Theorem 5.1. Let $M$ be a closed 5-manifold whose fundamental group has one end and $H^{2}(\pi, \mathbb{Z}[\pi])$ is either 0 or $\mathbb{Z}$. If $M$ admits a metric with zero entropy, then:

(1) $\operatorname{dim} H_{2}(\tilde{M}, \mathbb{Q}) \leq 1$ and $H_{2}(\tilde{M})$ has no finite subgroup as a direct summand;

(2) $H_{3}(\tilde{M})$ is either 0 or $\mathbb{Z}$;

(3) if $H_{3}(\tilde{M})=\mathbb{Z}$, then $H_{2}(\tilde{M})$ is a torsion group with no finite subgroup as a direct summand.

An example of a group as in item 3 is the quasicyclic group of type $p^{\infty}$ ( $p$ prime) given by the $p^{k}$-th roots of unity, $k$ running over all natural integers. We do not know if such a group can be realized as $\pi_{2}(M)$ of a 5 -manifold.

Proof. Since the fundamental group of $M$ has one end, $H_{4}(\tilde{M})=0$. By Theorem C, $H_{3}(\widetilde{M})$ is 0 or $\mathbb{Z}$ and we can apply Proposition 3.4 to $\widetilde{M}$. This proposition combined with the Technical Lemma proves all the claims in the theorem except the one regarding the absence of finite groups of $H_{2}(\widetilde{M})$ as a direct summand. Suppose there 
is such a group. Then there exists a prime $p$ such that the group $\mathbb{Z}_{p^{k}}$ appears as a direct summand for some $k \geq 1$. By Proposition 3.2, $\widetilde{M}$ dominates the Moore space $M\left(\mathbb{Z}_{p^{k}}, 2\right)$, but the latter has the property that its loop space homology with coefficients in $\mathbb{Z}_{p}$ grows exponentially. Again, this cannot happen by the Technical Lemma.

Note added in proof. J. A. Hillman pointed out to us that in Corollary 4.4 (and thus also in Theorems A and 4.5) "s-cobordant" can be replaced by "homeomorphic", see Theorem 10.10 in [12].

\section{References}

[1] J. W. Anderson, G. P. Paternain, The minimal entropy problem for 3-manifolds with zero simplicial volume. Geometric methods in dynamics (I), Asterisque 286 (2003), 63-79. Zbl 1056.53054 MR 2052297

[2] M. Aubry, Homotopy theory and models. based on lectures held at a DMV seminar in Blaubeuren by H. J. Baues, S. Halperin and J.-M. Lemaire. DMV Seminar 24, Birkhäuser, Basel 1995. Zbl 0854.55001 MR 1347447

[3] E. I. Dinaburg, On the relations among various entropy characteristics of dynamical systems. Math. USSR Izv. 5 (1971), 337-378. Zbl 0248.58007

[4] B. Eckmann, Amenable groups and Euler characteristic. Comment. Math. Helv. 67 (1992), 383-393. Zbl 0765.57019 MR 1171301

[5] B. Eckmann, Manifolds of even dimension with amenable fundamental group. Comment. Math. Helv. 69 (1994), 501-511. Zbl 0857.57002 MR 1303224

[6] Y. Félix, S. Halperin, J. C. Thomas, Rational homotopy theory. Grad. Texts in Math. 205, Springer-Verlag, New York 2001. Zbl 0961.55002 MR 1802847

[7] M. Gromov, Homotopical effects of dilatations. J. Differential Geom. 13 (1978), 303-310. Zbl 0427.58010 MR 0551562

[8] M. Gromov, Groups of polynomial growth and expanding maps. Inst. Hautes Études Sci. Publ. Math. 53 (1981) 53-73. Zbl 0474.20018 MR 0623534

[9] M. Gromov, Volume and bounded cohomology. Inst. Hautes Études Sci. Publ. Math. 56 (1982), 5-99. Zbl 0516.53046 MR 0686042

[10] M. Gromov, Metric structures for Riemannian and non-Riemannian spaces. Translated from the French by S. M. Bates, Progr. Math. 152, Birkhäuser, Boston, MA, 1999. Zbl 0953.53002 MR 1699320

[11] A. Hatcher, Algebraic Topology. Cambridge University Press, Cambridge 2002. Zbl 1033.55001 MR 1867354

[12] J. A. Hillman, Four-manifolds, geometries and knots. Geometry and Topology Monographs 5, Geometry and Topology Publications, Coventry, 2002. Zbl 01872947 MR 1943724 
[13] P. Hilton, G. Mislin, J. Roitberg, Localization of nilpotent groups and spaces. NorthHolland Mathematics Studies 15, North-Holland Publishing Co., Amsterdam, Oxford; American Elsevier Publishing Co., Inc., New York 1975. Zbl 0323.55016 MR 0478146

[14] R. Mañé, On the topological entropy of geodesic flows. J. Differential Geom. 45 (1997), 74-93. Zbl 0896.58052 MR 1443332

[15] A. Manning, Topological entropy for geodesic flows. Ann. of Math. 110 (1979), 567-573 Zbl 0426.58016 MR 0554385

[16] G. P. Paternain, Geodesic Flows. Prog. Math. 180, Birkhäuser, Boston, MA, 1999. Zbl 0930.53001 MR 1712465

[17] G. P. Paternain, J. Petean, Minimal entropy and collapsing with curvature bounded from below. Invent. Math. 151 (2003), 415-450. Zbl 1049.53029 MR 1953264

[18] G. P. Paternain, J. Petean, Entropy and collapsing of compact complex surfaces. Proc. London Math. Soc. 89 (2004), 763-786. Zbl 02152574 MR 2107014

[19] Y. Yomdin, Volume growth and entropy. Israel J. Math. 57 (1987), 285-300. Zbl 0641.54036 MR 0889979

Received July 19, 2004

Gabriel P. Paternain, Department of Pure Mathematics and Mathematical Statistics, University of Cambridge, Cambridge CB3 0WB, England

E-mail: g.p.paternain@dpmms.cam.ac.uk

Jimmy Petean, CIMAT, A.P. 402, 36000, Guanajuato. Gto., México

E-mail: jimmy@ cimat.mx 